\title{
El papel del maestro desde el humanismo psicológico. Una mirada epistemológica
}

\author{
The role of the teacher from the psychological humanism. An epistemological view
}

\section{José Nava}

Instituto Superior de Ciencias de la Educación del Estado de Mexico - México

Toluca, México

nava5812@yahoo.com.mx

\section{RESUMEN}

El objetivo del artículo consistió en encontrar la relación entre las facultades cognoscitivas del sujeto cognoscente y los problemas del conocimiento en la construcción de los argumentos sobre el papel del maestro desde la teoría psicológica humanista aplicada a la educación. Se utilizó como metodología el análisis epistemológico del discurso descifrando los sentidos y significados gnoseológicos de las ideas, juicios y raciocinios empleados por los teóricos de dicha corriente en la edificación mencionada, buscando comprender las facultades cognoscitivas con las que se pretenden resolver los problemas filosóficos del conocimiento en la misma. Los resultados revelan la existencia de una relación o correspondencia de dependencia de las facultades cognoscitivas del sujeto cognoscente respecto de determinados problemas del conocimiento desde los que se construye la propuesta desde dicha corriente psicológica. Finalmente, se concluye que detrás de la propuesta analizada, se encuentran ciertas facultades cognoscitivas que determinan la manera en que se pretenden resolver los principales problemas del conocimiento en la construcción del concepto estudiado.

Palabras Clave: facultades cognoscitivas; supuestos filosóficos; relación; humanismo psicológico; papel del maestro

\section{ABSTRACT}

The objective of the article was to find the relationship between the cognitive faculties of the cognitive subject and the problems of knowledge in the construction of the arguments on the role of the teacher from the humanistic psychological theory applied to education. The methodology used was the epistemological analysis of the discourse, deciphering the gnoseological senses and meanings of the ideas, judgments, and reasoning used by the theoreticians of this current in the mentioned edification, seeking to understand the cognitive faculties with which they intend to solve the philosophical problems of knowledge in it. The results reveal the existence of a relationship or correspondence of dependence of the cognitive faculties of the cognitive subject with respect to certain problems of knowledge from which the proposal is built from the said psychological current. Finally, it is concluded that behind the analyzed proposal, there are certain cognitive faculties that determine the way in which the main problems of knowledge are intended to be solved in the construction of the studied concept.

Keywords: cognitive faculties; philosophical assumptions; relationship; psychological humanism; teacher's role 


\section{Introducción}

Los conceptos pedagógicos como las metas de la educación, el concepto de aprendizaje, el papel del maestro, el concepto de alumno, la motivación, la metodología de la enseñanza, la evaluación, etc., de las teorías educativas (conductismo, psicoanálisis, humanismo, cognoscitivismo, teoría genética de Piaget, teoría socio-cultural de Vygotsky, etc.) no son obra de la casualidad, sino que obedecen a ciertas relaciones onto-epistemológicas de carácter meta-científico entre determinadas necesidades existenciales y ontológicas de los sujetos cognoscentes, los problemas filosóficos del conocimiento, las facultades cognoscitivas con los que los investigadores pretenden resolverlos, los supuestos filosóficos en los que necesariamente se ubican al hacerlo, los principios de la ciencia y el discurso educativo. Según Ortega y Gasset (1998, p. 21):

Toda idea es pensada y todo cuadro es pintado desde ciertas suposiciones o convenciones tan básicas, tan de clavo pasado para el que pensó la idea o pintó el cuadro, que ni siquiera repara en ellas y por lo mismo no las introduce en su idea ni en su cuadro, no las hallamos allí puestas sino precisamente supuestas y como dejadas a la espalda. Por eso, a veces, no entendemos una idea o un cuadro: nos falta la palabra del enigma, la clave de la secreta convicción.

A ello se debe que los conceptos mencionados sean contradictorios entre sí y que no sea posible determinar cuáles de ellos son los verdaderos y cuales sean los falsos, los buenos o los malos, los mejores o los peores, etc. Es decir, la ciencia no es neutral, obedece a determinadas necesidades ontológicas y epistemológicas derivadas de la problemática existencial del ser humano.

Como resultado de las investigaciones epistemológicas y de los seminarios que se imparten sobre la materia en los posgrados en educación en diferentes instituciones públicas y privadas, se ha podido observar lo que parecen ser algunas relaciones entre las facultades cognoscitivas del sujeto cognoscente (Polo, 2006; Verneaux, 2011) y los problemas del conocimiento (Hessen, 1999) en la construcción de saberes en el campo educativo.

Tal es el caso de la teoría psicológica humanista aplicada a la educación (Guzmán y Hernández, 1993). Si se analizan con cuidado los mapeos del papel del maestro elaborados a partir de los supuestos del humanismo psicológico (Rogers, 1991; Rogers y Freiberg, 1996), es posible prestar atención a una serie de relaciones entre las facultades cognoscitivas del sujeto cognoscente y los problemas del conocimiento.

En este estudio, se parte de la creencia de que el sujeto cognoscente -con conocimiento o no de este hecho- utiliza determinadas facultades cognoscitivas para elaborar el mapeo sobre el papel del maestro desde la teoría psicológica humanista aplicada a la educación, que se relacionan con determinados problemas del conocimiento. Es decir, que se puede establecer una correspondencia directamente proporcional entre las facultades cognoscitivas del sujeto cognoscente y los problemas del conocimiento. A cada facultad cognoscitiva corresponde determinado problema del conocimiento.

Debido a lo anterior, el objetivo de la presente investigación consistió en comprender con qué tipo de facultades cognoscitivas se pretenden resolver los problemas del conocimiento en la construcción del mapeo sobre el papel del maestro desde el humanismo psicológico aplicado a la educación. 


\section{Metodología}

El artículo se elaboró utilizando como metodología el análisis epistemológico del discurso que consiste en descifrar los sentidos y significados gnoseológicos de las ideas, juicios y raciocinios empleados por los teóricos de la ciencia cuando pretenden construir conocimiento sobre cualquier realidad. Aplicando dicha metodología se compararon las facultades cognoscitivas del sujeto cognoscente de la teoría psicológica humanista con los problemas del conocimiento, en la construcción de los argumentos sobre el papel que debe desempeñar el docente en el proceso enseñanza-aprendizaje.

En un primer momento, se construyó la idea sobre los problemas del conocimiento (posibilidad, origen, esencia, clasificación y criterios de verdad) a los que se enfrenta el sujeto cognoscente cuando pretende hacer ciencia, a partir de autores como Polo (2006), Hessen (2009), Vermeaux (2011), entre otros.

En segundo lugar, se construyó la idea sobre las facultades cognoscitivas (racionales y/o sensuales) con las que el sujeto cognoscente de la teoría psicológica humanista pretende relacionarse con su objeto de estudio, cuando intenta realizar mapeos sobre el papel del maestro en el proceso enseñanza aprendizaje; desde la mirada de autores como Polo (2006), Platón (2001), Descartes (2018), Spinoza (1990), Schopenhauer (1997), Nietzsche (1976), Kuhn (1999), James (1975), Kant (1994), entre otros.

Finalmente, se estableció la relación entre las facultades cognoscitivas del sujeto cognoscente de la teoría psicológica humanista y los problemas del conocimiento a los que se enfrenta, cuando pretende hacer ciencia psicológica aplicada a la educación.

\section{Desarrollo.}

\subsection{Los problemas del conocimiento.}

El conocimiento humano se puede entender como un proceso en el que se relacionan un sujeto cognoscente y un objeto por conocer. Esto quiere decir que los elementos esenciales de todo proceso de conocimiento son el sujeto cognoscente, el objeto por conocer y la relación que debe establecerse entre ellos para que se dé el conocimiento.

Antes de establecerse la relación de conocimiento, ambos elementos, tanto el sujeto como el objeto, sólo son entes, seres que existen independientemente el uno del otro. Ambos se encuentran en la esfera ontológica, en la realidad, que puede ser concreta o abstracta.

Se estima que el objeto de conocimiento surge en tanto que un ente (en este caso se supone que únicamente el ser humano es capaz de conocer) fija su atención en otro ente cualquiera (material o inmaterial) con la intención de conocerlo porque "la objetividad se convierte con la intencionalidad precisamente porque el objeto conocido no se da de suyo" (Polo, 2006, p. 41) y, a su vez, el ser humano que fijo su atención en otro ente con miras a conocerlo, de simple ente que era, antes de relacionarse con el objeto para conocerlo, se transforma en sujeto cognoscente al fijar su atención en un objeto para entenderlo; “el conocimiento es un acto, espontáneo en cuanto a su origen, inmanente en cuanto a su término, por el que un hombre se hace intencionalmente presente alguna región del ser" (Verneaux, 2011, pp. 103-104). Es la intención de conocer la que 
hace posible el surgimiento de conceptos como 'sujeto' y 'objeto' de conocimiento. Eso permite suponer que todo conocimiento que se pretenda construir será interesado. En ese entendido, la ciencia no es neutral, parece ser que obedece a intereses personales y/o de grupo.

El problema del conocimiento surge cuando el sujeto pretende establecer la relación de discernimiento con el objeto, debido a que ambos (el sujeto cognoscente y el objeto por conocer) se encuentran en mundos diferentes, distintos y, hasta contrarios: el sujeto cognoscente es el alma humana, su psique, pensamiento, razón, mente, etc., y por lo mismo, se encuentra en la esfera psicológica. En cambio, el objeto por conocer es la realidad (que puede ser material o inmaterial), pertenece a la esfera ontológica. Hessen afirma que "el conocimiento se presenta como una relación entre estos dos miembros - se refiere al sujeto y al objeto -, que permanecen en ella eternamente separados el uno del otro" (2011, p. 15). Lo que permite estimar que no es posible conocer. Cuando el sujeto cognoscente (el alma humana) pretende traspasar la barrera del objeto por conocer (la realidad concreta o abstracta) para aprehender literalmente al objeto por comprender, choca, también literalmente, contra una barrera que le impone la realidad. Son mundos, planos o esferas diferentes en las que existen el sujeto y el objeto; y, por eso, no se pueden fusionar.

En virtud de que el conocimiento de la realidad es imposible, surge el problema del conocimiento "el espíritu no puede salir de sí mismo para coincidir con las cosas...una cosa no puede entrar en el espíritu" (Verneaux, 2011, p. 77). Ni la conciencia cognoscente puede salir de sí misma para penetrar la esfera del objeto, ni éste puede entrar en la mente.

Dice Hessen que,

...vista desde el sujeto, esta aprehensión se presenta como una salida del sujeto fuera de su propia esfera, una invasión en la esfera del objeto y una captura de las propiedades de éste. El objeto no es arrastrado, empero, dentro de la esfera del sujeto, sino que permanece trascendente a él (2009, p. 16).

Es decir, para el sujeto cognoscente (el alma humana) le es imposible penetrar la esfera del objeto por conocer (la realidad concreta o abstracta).

Para Verneaux,

...el objeto y el sujeto son definibles únicamente por su relación mutua que es el conocimiento. ¿Qué es un objeto, una cosa, un ser? Lo que aparece a un sujeto. ¿Qué es un sujeto, una conciencia, un espíritu? Aquello en quien o a quien aparece un objeto (2011, p. 72$)$.

Por lo tanto, la realidad (concreta o abstracta) trasciende al sujeto cognoscente, es decir, está fuera de él, en otro mundo.

A partir de lo desarrollado en los párrafos anteriores, es posible suponer las razones a las que se debe que los grandes problemas del conocimiento, como son su posibilidad, origen, esencia, clasificación, conceptos y criterios de verdad, entre otros, no han sido resueltos de manera definitiva a lo largo de la historia de la filosofía. 
3.2 Las facultades cognoscitivas con las que el sujeto cognoscente se relaciona con su objeto de estudio.

Si estamos de acuerdo en que el conocimiento de la realidad es imposible en esencia, tenemos que determinar de alguna manera cómo es que se establece la relación de conocimiento entre el sujeto cognoscente y el objeto por conocer y qué tipo de relación es ésta.

Se puede presumir que el sujeto cognoscente es el que toma la iniciativa para relacionarse con su objeto de conocimiento mediante determinadas acciones voluntarias: "el conocimiento, en cuanto tal, es acto, y, al menos, ese acto es operación: a la operación corresponde un 'objeto' la operación supone una facultad" (Polo, 2006, p. 15). Se presume que esa facultad o, más bien facultades, pueden referirse al alma y al cuerpo humanos:

Gracias a su situación intermedia, es el alma portadora de los caracteres de ambos mundos; hay en ella algo privativo del mundo de las Ideas y algo peculiar del de la percepción. Lo primero es la racionalidad (logistikón o nous), la morada del saber y de su correspondiente virtud. En lo segundo, lo irracional, distingue Platón dos cosas: lo más noble y vuelto hacia la razón y lo más insano y alejado de ella. Lo más noble reside en la fuerza volitiva (entusiasmo, thimós); lo insano, en la apetencia sensorial (impulso, epithymía). Según esto, razón, entusiasmo e impulso son las tres actividades del alma, las tres formas (eidée) de sus posibles estados (Platón, 2001, p. XXIII).

Según lo anterior, la necesidad que satisface el alma humana, en términos existenciales, consiste en generar ideas sobre la realidad (lo racional); mientras que el cuerpo se encarga de la percepción (lo irracional). En lo que se refiere a la relación de conocimiento:

...no hay mejor camino para llegar al conocimiento de nuestras pasiones que examinar la diferencia existente entre el alma y el cuerpo, a fin de conocer a cuál de los dos se debe atribuir cada una de las funciones que hay entre nosotros (Descartes, 2018, p. 6).

Las funciones del alma y del cuerpo difieren, pues "ni el cuerpo puede determinar el alma a pensar, ni el alma puede determinar al cuerpo al movimiento o al reposo o a alguna otra manera de ser" (Spinoza, 1990, p. 72). Se puede estimar que el alma se encarga de lo inmaterial o espiritual y el cuerpo de lo perceptible o sensible. Dos facultades cognoscitivas por medio de las cuales el sujeto cognoscente se relaciona con el objeto por conocer; una, produce ideas (el alma); la otra, percibe lo material (el cuerpo).

Se entiende que todo conocimiento es un proceso en el que un sujeto cognoscente (facultad) se relaciona con un objeto por conocer (realidad) mediante determinados actos, operaciones o acciones voluntarias sobre su objeto de conocimiento que realiza con su razón y sus sentidos.

Si los sujetos cognoscentes sólo cuentan con su razón y sus sentidos para relacionarse con los objetos por conocer, en todas y cada una de las posibilidades de realización del espíritu humano (ciencia, arte, religión y filosofía), sólo tienen, en un primer momento -matemáticamente hablando-, dos posibilidades para establecer dicha correspondencia: la razón y los sentidos.

En un segundo momento, es posible combinar las dos facultades mencionadas. En este caso, el sujeto cognoscente cuenta con otras dos posibilidades para relacionarse con su objeto de estudio: utilizar primero la razón y después los sentidos o viceversa. 
Parece ser que -de manera fatal- el sujeto cognoscente solo cuenta con cuatro y sólo cuatro posibilidades de relacionarse con su objeto de estudio: la razón, los sentidos, la razón primero y los sentidos después o los sentidos primero y posteriormente la razón.

Si la realidad u objeto por conocer se aprecia con las facultades cognoscitivas del sujeto cognoscente y si éstas se reducen a dos y a su combinación: ¿qué intensiones, intereses o motivos del sujeto cognoscente le conducen a utilizar alguna de esas facultades o su combinación?

Parece ser que esas intenciones, intereses o motivos del sujeto cognoscente se refieren a conceptos, nociones, categorías o ideas que tienen que ver con los términos o palabras que se refieren a lo racional, sensual, racional-sensual y sensual-racional. Lo racional implica orden, lo sensual, caos; lo racional envuelve determinismo, lo sensual libertad o indeterminismo; lo racional involucra ideas, lo sensual la realidad; lo racional comprende dualismos, lo sensual unidades; lo racional incluye quietud, lo sensual movimiento o cambio; lo racional permite un conocimiento mediato, lo sensual un conocimiento inmediato; lo racional es inmanente, lo irracional trascendente; lo racional-sensual y lo sensual racional, respectivamente, implican estados intermitentes entre uno y otro de las mencionadas intensiones, intereses o motivos .

Es decir, las facultades cognoscitivas del sujeto cognoscente le permiten a éste apreciar al objeto por conocer en distintas acciones, pasiones o estados, dependiendo de qué facultad cognoscitiva se utilice para relacionarse con el objeto por conocer y dicha facultad, su vez, depende de sus motivos, intensiones o intereses existenciales.

En este caso concreto: ¿cuál es la facultad o facultades cognoscitivas con las que el sujeto cognoscente de la teoría humanista aplicada a la educación se relaciona con su objeto de estudio y cuáles son sus alcances y limitaciones en cuanto al conocimiento sobre el papel del maestro en el proceso enseñanza-aprendizaje?

Para resolver la cuestión anterior, parece que basta, a manera de ejemplo, analizar el mapeo que -sobre el papel del maestro- se realiza desde la psicología humanista aplicada a la educación. Desde la psicología humanista aplicada a la educación se argumenta lo siguiente sobre el papel del maestro:

El núcleo central del papel del docente en una educación humanista está basado en una relación de respeto con sus alumnos. El profesor debe partir siempre de las potencialidades y necesidades individuales de los estudiantes y con ello fomentar un clima social para que sea exitosa la comunicación de la información académica y emocional (Hamachek, 1987; citado por Guzmán y Hernández, 1993, p. 46).

Si relacionamos la cita anterior con las facultades cognoscitivas con las que el sujeto cognoscente pretende relacionarse con su objeto de estudio, es posible apreciar que los términos potencialidades y necesidades individuales tienen un origen sensual; es decir, implican caos o desorden, en el sentido de que todos y cada uno de los alumnos cuentan con potencialidades y necesidades diferentes e, incluso, contradictorias entre sí y con relación a los demás.

El papel del maestro se interpreta como un facilitador del aprendizaje proporcionando las condiciones para que se dé en forma autónoma, partiendo de las potencialidades y necesidades individuales y fomentando un clima social (Guzmán y Hernández, 1993, pp. 47-48). 
Esta perspectiva asigna al profesor un papel no directivo dentro del proceso de enseñanzaaprendizaje; postula que su función debe ser la de facilitar el aprendizaje del estudiante a través de proporcionarle las condiciones para que este acto se dé en forma autónoma (Guzmán, y Hernández, 1993, p. 47).

Es posible apreciar que los términos 'facilitadores del aprendizaje', 'autonomía del alumno para conducir su proceso enseñanza-aprendizaje', y otros más, tienen un origen subjetivo; es decir, sensual; dichas palabras implican indeterminismo o libertad en el sentido de que todos y cada uno de los alumnos cuentan con autonomía y libertad para conducir por sí mismos el proceso enseñanza-aprendizaje y sin dirección.

Se observa el papel del maestro de manera sensual y se llega al siguiente discurso: "El mecanismo para conseguir lo anterior es convertir a los salones de clase en comunidades de aprendizaje, donde la obtención de nuevos conocimientos recupere su sentido lúdico, placentero y libertario" (Guzmán y Hernández, 1993, p. 47). Los términos 'lúdico', 'placentero' y 'libertario’ permiten suponer que la relación entre las facultades cognoscitivas del sujeto cognoscente y las acciones, pasiones o estados del ser del ente se dan en términos de cambio o movimiento. Es decir, todos y cada uno de los sujetos del acto educativo sienten y piensan diferente, dependiendo de sus intereses, intenciones o motivos existenciales; que dependen de circunstancias de modo, tiempo y lugar.

En la siguiente cita también es posible apreciar la facultad cognoscitiva con la que el sujeto cognoscente se relaciona con su objeto de estudio "La sugerencia es crear una atmósfera total de respeto y apoyo a la curiosidad, la duda, valorar la búsqueda personalizada de los conocimientos, donde todas las cosas se vuelven objeto de estudio y exploración" (Guzmán y Hernández, 1993, p. 47). Los términos 'respeto', 'apoyo', 'curiosidad', 'duda', 'búsqueda personalizada', y otros más, parecen indicar que el sujeto cognoscente se está relacionando con su objeto de estudio utilizando sus sentidos como facultad cognoscitiva.

Lo anterior permite suponer que la realidad que se refiere al papel del maestro, dentro del proceso enseñanza- aprendizaje, se encuentra en constante movimiento o cambio en tiempo, espacio y circunstancias.

El sujeto cognoscente del humanismo aplicado a la educación sigue relacionándose con su objeto de estudio de manera sensual: "El profesor no dirige estas comunidades ni es alguien extraño, sino que participa como facilitador e integrante más del grupo” (Guzmán y Hernández, 1993, p. 47). Lo irracional está presente en esta cita. En ella reina el caos o desorden, en el sentido de que no existe quien pretenda dirigir el proceso enseñanza-aprendizaje.

Se dice que "por ello es importante que estimule y propicie la cooperación y apoyo entre compañeros sin que esperen recibir premios externos por ello" (Guzmán y Hernández, 1993, p. 47). Se entiende el papel del profesor como alguien que se debe adaptar a cada circunstancia de tiempo, modo y lugar de los alumnos:

La labor docente es considerada no como el uso o aplicación de técnicas didácticas para supuestamente lograr una enseñanza eficaz, sino modificar las actitudes de los maestros para que desempeñan en forma radicalmente distinta su trabajo, aceptando nuevas formas de enseñanza (Guzmán y Hernández, 1993, p. 48). 
Ello significa que lo sensual impera sobre lo racional, es decir, el desorden o caos somete al orden. Prevalece el movimiento o cambio sobre la quietud: "Los humanistas sostienen que sin un cambio de las actitudes y creencias de los profesores los efectos de cualquier innovación didáctica serán sólo de corto plazo o inexistentes" (Guzmán y Hernández, 1993, p. 48).

Es decir, las facultades cognoscitivas del teórico del humanismo psicológico aplicado a la educación le permiten a éste apreciar el papel del maestro en determinadas acciones, pasiones o estados de movimiento y/o cambio, caos o desorden, indeterminismo o libertad, existencia concreta (realismo), unidad o inclusión, intuitivamente y de manera trascendente, a causa de que la facultad cognoscitiva que se utiliza para relacionarse con el objeto por conocer lo son sus sentidos.

\subsection{Las facultades cognoscitivas del sujeto cognoscente y los problemas del conocimiento.}

Es posible establecer una relación de causa efecto entre las facultades cognoscitivas del sujeto cognoscente y los problemas del conocimiento en la construcción del discurso sobre el papel del maestro, desde la mirada de la psicología humanista aplicada a la educación.

Se estima que a cada problema del conocimiento corresponden cuatro posibilidades de relación con las facultades cognoscitivas del sujeto cognoscente: la razón, los sentidos, la razón primero y los sentidos después o los sentidos primero y la razón después. En ese entendido, a cada facultad cognoscitiva del sujeto cognoscente corresponden determinadas acciones, pasiones o estados del ser del ente dependiendo del problema del conocimiento con el que se relacione la facultad cognoscitiva del sujeto cognoscente. Esto sucede con independencia de que el sujeto cognoscente esté o no consciente de tal hecho.

En el caso particular de la psicología humanista aplicada a la educación, es posible observar que, para construir el mapeo del papel del maestro en el proceso enseñanza-aprendizaje, el sujeto cognoscente pretende solucionar los problemas del conocimiento utilizando -con conocimiento o no de este hecho- determinada facultad cognoscitiva que obedece a sus necesidades, propósitos o intenciones existenciales, más que a una solución esencial y verdadera. Esto se podrá observar en los siguientes apartados.

\subsubsection{El problema de la posibilidad del conocimiento y las facultades cognoscitivas del sujeto cognoscente.}

La psicología humanista estima que, para explorar, describir, explicar, interpretar o comprender el papel del maestro en el proceso enseñanza-aprendizaje, se debe comenzar por aceptar que éste se encuentra en constante cambio o movimiento de un sujeto a otro: "He llegado a sentir que sólo existe una persona (al menos mientras yo viva, y quizá también después) capaz de saber si lo que hago es honesto, o bien es falso, hipócrita o incoherente: esa persona soy yo" (Rogers, 1991, p. 32). Es decir, se supone que cada maestro siente y piensa diferente a los demás y que,

...no hay otra verdad más cierta, más independiente ni que necesite menos pruebas de la de que todo lo que puede ser conocido, es decir, el universo entero, no es objeto más que para un sujeto, percepción del que percibe; en una palabra: representación (Schopenhauer 1997). 
Si estamos de acuerdo con lo anterior, entonces, es posible inferir que esas acciones, pasiones o estados de del ser del ente que se suponen (cambio o movimiento) sobre el papel del maestro en el proceso enseñanza-aprendizaje se pueden relacionar con el problema de la posibilidad del conocimiento: ¿es posible conocer el papel del maestro en el proceso enseñanza aprendizaje? No, en esencia, porque este consiste, para la psicología humanista aplicada a la educación, en "liberar la curiosidad, permitir que las personas evolucionen según los propios intereses, desatar el sentido de indagación, abrir todo a la pregunta y la exploración, reconocer que todo está en proceso de cambio, aunque nunca lo logre de manera total" (Rogers y Freiberg, 1996, p. 184). Es decir, el papel del maestro en el proceso enseñanza aprendizaje se encuentra en un estado del ser que implica evolución, intereses subjetivos, proceso de cambio, entre otros. O sea, se trata de una acción, pasión o estado del papel del maestro en el proceso enseñanza-aprendizaje, que se relaciona muy bien con los sentidos:

Veo la facilitación del aprendizaje como el objetivo de la educación, como el modo de formar al hombre que aprende, el modo de aprender a vivir como individuos en evolución. La facilitación del aprendizaje es una actividad que puede formular respuestas constructivas, cambiantes y flexibles a algunos de los problemas más profundos que acosan al hombre moderno (Rogers y Freiberg, 1996, p. 185).

La idea 'individuos en evolución', denota estados del papel del maestro en el proceso enseñanzaaprendizaje de movimiento o cambio y se puede relacionar con los sentidos del sujeto cognoscente y, a su vez, éstos se relacionan con el problema de la posibilidad del conocimiento.

Es posible concluir, en este caso, que el problema de la posibilidad del conocimiento se encuentra directamente relacionado con los sentidos del sujeto cognoscente en la construcción del mapeo sobre el papel del maestro en el proceso enseñanza-aprendizaje, desde la mirada de la psicología humanista aplicada a la educación. A las nociones, ideas, categorías, o conceptos como ‘evolución', 'intereses', 'proceso de cambio', entre otras, corresponden estados del ser del ente relacionados con el cambio o movimiento de la realidad del maestro en el proceso enseñanza-aprendizaje, lo que se lleva muy bien con el problema de la posibilidad del conocimiento.

\subsubsection{El problema del origen del conocimiento y las facultades cognoscitivas del sujeto cognoscente.}

La psicología humanista aplicada a la educación sostiene que para comprender el papel del maestro en el proceso enseñanza-aprendizaje se debe partir del supuesto de que no es posible enseñar a enseñar: "Mi experiencia me dice que no puedo enseñar a otra persona cómo enseñar" (Rogers, 1999, p. 243). El término 'experiencia' indica práctica, usanza, estilo, rutina, uso, etc., es decir, se supone que la enseñanza de la enseñanza es un arte empírico, práctico, materialista, efectivo, real, etc. Si el papel del profesor no es el de enseñar, entonces en el aprendizaje reina el caos o desorden, el desconcierto, la anarquía; es decir, la falta de dirección, orientación, trayectoria, itinerario, consejo, etc. Ese desorden o caos permite estimar que la enseñanza del maestro es intrascendente y no influye la conducta del alumno: "Pienso que cualquier cosa que pueda enseñar a otra persona es relativamente intrascendente y ejerce poca o ninguna influencia sobre la conducta" (Rogers, 1999, p. 243). El término 'intrascendente' indica lo ligero, baladí, trivial o frívolo, es decir, insignificante. 
Por ello, se supone que el aprendizaje debe ser descubierto e incorporado por el propio alumno: "He llegado a sentir que el único aprendizaje que puede influir significativamente sobre la conducta es el que el individuo descubre e incorpora por sí mismo" (Rogers, 1999, p. 243). Los términos 'descubrimiento' e 'incorporación’ envuelven sentidos y significados como hallazgo y afiliación, respectivamente, "la independencia es el privilegio de los fuertes, de la reducida minoría que tiene el valor de autoafirmarse" Nietzsche (1976, p. 41). De acuerdo con lo anterior, el aprendizaje debe ser encontrado por el propio alumno: “el aprendizaje basado en el propio descubrimiento, la verdad incorporada y asimilada personalmente en la experiencia, no puede comunicase de manera directa a otro" (Rogers, 1999, p. 243).

El único papel que se le otorga al maestro, desde la mirada de la psicología humanista aplicada a la educación, es el de facilitador: "El docente facilitador comparte con otros -con los estudiantes y quizá también con los padres o los miembros de la comunidad- la responsabilidad del proceso de aprendizaje" (Rogers, 1999, p. 246). El término 'facilitador' se pude interpretar como un apoyo técnico con el que el alumno puede contar para construir sus propios saberes sobre la realidad. "El facilitador suministra recursos para el aprendizaje, procedente de él mismo y de sus experiencias, de libros, o materiales, o experiencias de la comunidad" (Rogers y Freiberg, 1996, p. 247). El papel del profesor es suministrar sus experiencias o prácticas, usos, estilos, rutinas, etc. sobre la adquisición de conocimientos para que el alumno las considere dependiendo de sus intereses. El papel del profesor como facilitador le convierte en un recurso más del proceso enseñanza-aprendizaje porque "el estudiante desarrolla su propio programa de aprendizaje, sólo o en colaboración con otros” (Rogers y Freiberg, 1996, p. 247).

En apoyo a lo anterior, otro de los papeles del maestro es:

...crear una atmósfera total de respeto y apoyo a la curiosidad, la duda, valorar la búsqueda personalizada de los conocimientos, donde todas las cosas se vuelvan objeto de estudio y valoración. El profesor no dirige estas comunidades ni es alguien extraño, sino que participa como un facilitador e integrante más del grupo (Guzmán y Hernández, 1993, p. 47).

Es posible concluir que el problema del origen del conocimiento se encuentra directamente relacionado con las facultades cognoscitivas del sujeto cognoscente (los sentidos) en la construcción del mapeo sobre el papel del maestro en el proceso enseñanza-aprendizaje, desde la mirada de la psicología humanista aplicada a la educación, en el sentido de que se está promoviendo el caos, el desconcierto, la anarquía, la desorganización, el desorden, entre otros. Es decir, que "de los sentidos proviene toda manifestación de certidumbre, toda buena conciencia, toda evidencia de verdad" Nietzsche (1976, p. 88). Se estima que las ideas, categorías, nociones o conceptos como 'experiencia', 'intrascendencia', 'incorporación', 'facilitador', entre otros, se relacionan muy bien con el caos o desorden. A su vez, el caos o desorden son los estados del ser del ente o principios del conocimiento que hacen posible estimar que el conocimiento se origina en los sentidos. Si se supone que la fuente y base del conocimiento son los sentidos, entonces, de alguna manera, la facultad cognoscitiva (los sentidos) que permite ver esto se relaciona muy bien con el problema del origen del conocimiento. 
3.3.3 El problema de la relación sujeto-objeto de conocimiento y las facultades cognoscitivas del sujeto cognoscente.

La psicología humanista aplicada a la educación sostiene que para comprender el papel del maestro en el proceso enseñanza-aprendizaje se debe partir del supuesto de que éste debe dejar en absoluta libertad al alumno para que construya su propio aprendizaje a partir de sus propias necesidades de conocimiento, suponiendo que, al hacer esto, "cada uno de ellos se convertirá en un estudiante con mayor capacidad de iniciativa, más original y auto disciplinado, menos ansioso, y disminuirá su tendencia a ser dirigido por los otros" (Rogers, 1999, p. 44). Los términos 'iniciativa', 'originalidad', 'autodisciplina', 'no dirección', entre otros, indican independencia, emancipación, autonomía, entre muchos más que denotan indeterminismo o libertad. Se opina que el maestro debe aceptar al alumno como éste es, el cliente "debe experimentar o percibir, al menos en cierta medida, la coherencia, aceptación y empatía del terapeuta” (Rogers, 1999, p. 251). Las palabras ‘coherencia’, ‘aceptación’ y ‘empatía’ suponen conexión, aprobación y tolerancia, respectivamente e indican que el maestro comprende al alumno, es decir, que se pone en su lugar. Que el maestro sea coherente y empático, implica, entre otras cosas, que “a) es auténtico e internamente consecuente consigo mismo; b) acepta al cliente y lo aprecia como persona de valor; c) comprende de manera empática al mundo privado de sentimientos y actitudes del cliente" (Rogers, 1999, pp. 324-325).

Se conjetura que el maestro es un recurso más del proceso enseñanza-aprendizaje a disposición de las necesidades del alumno y que sus intenciones deben ser "que sus alumnos sepan que él posee conocimientos y una experiencia especial en un campo de estudio determinado y que tales recursos se hallan a disposición de ellos" (Rogers, 1999, p. 254). Se presume que un alumno que tiene a su disposición los recursos de la maestría es un alumno indeterminado, libre, independiente y autónomo respecto de su mentor; cuya función se concentrará en "aportar los recursos con que el estudiante puede aprender a cumplir esas exigencias" (Rogers, 1999, p. 256). se cree que "los alumnos aprenden más acerca de las cuestiones básicas, y, asimismo, exhiben la mayor creatividad y capacidad para resolver problemas" (Rogers y Freiberg, 1996, p. 27). Porque se admite que el maestro debe tener "confianza en la capacidad de cada alumno en particular" (Rogers y Freiberg, 1996, p. 134). Se sospecha que el maestro debe considerar que "su papel es el de invitar a los demás a aprender y a participar en el aprendizaje” (Rogers y Freiberg, 1996, p. 144). Porque se supone que: "los individuos necesitan experimentar la libertad de aprender por sí mismos, lo que, a su vez, les capacita para ejercer una labor facilitadora sobre otros" (Rogers y Freiberg, 1996, p. 152).

Las expresiones como 'iniciativa, 'originalidad', 'autodisciplina', 'no dirección', 'aceptación', 'empatía', 'confianza', 'creatividad', 'capacidad' y, muchos otros más, involucran sentidos de indeterminismo y libertad del alumno respecto de su maestro y del mismo sistema educativo y se pueden relacionar con el problema de la relación sujeto-objeto de conocimiento en el sentido de que, en ésta conexión, el sujeto cognoscente, es decir, el alumno, es el que determina al objeto por conocer (el conocimiento); es decir, el sujeto determina al objeto, en la relación de conocimiento entre los dos elementos mencionados.

Solo cuando el profesor tiene la capacidad de comprender desde dentro las reacciones del estudiante, "cuando tiene una percepción sensible de cómo se presenta el proceso de aprendizaje 
al alumno, entonces podrá facilitar un aprendizaje significativo" (Rogers y Freiberg, 1996, p. 189), constituyéndose en un dispensador de recursos:

se concentrará en proporcionar toda clase de recursos para que el aprendizaje sea vivencial y adecuado a las necesidades de los estudiantes. También tratará de que los recursos estén disponibles sin tener que pasar por trámites complicados para conseguirlos y simplificará los pasos prácticos y psicológicos necesarios para utilizarlos (Rogers y Freiberg, 1996, p. 218).

El maestro es un recurso más al servicio de las necesidades del alumno:

el recurso más importante es el propio maestro. Él mismo y sus conocimientos se ponen a disposición inmediata del alumno, pero sin imponerse. El maestro demuestra en que terreno en particular es más competente, y sus alumnos le pueden consultar en todo lo que él pueda ofrecer. Pero él se ofrece como recurso y el aprovechamiento de su capacidad dependerá de los estudiantes (Rogers y Freiberg, 1996, p. 219).

Es posible concluir que el problema de la relación sujeto-objeto de conocimiento se encuentra directamente relacionado con las facultades cognoscitivas del sujeto cognoscente (los sentidos) en la construcción del mapeo sobre el papel del maestro en el proceso enseñanza-aprendizaje, desde la mirada de la psicología humanista aplicada a la educación, en el entendido de que se está promoviendo la libertad o indeterminismo del alumno. Se estima que las ideas, categorías, nociones o conceptos como 'facilitador', 'confianza', 'no dirección', 'aceptación', 'empatía', 'aprobación', 'tolerancia', entre otros atributos que la teoría solicita del profesor, se relacionan muy bien con la libertad o el indeterminismo del alumno y del grupo escolar, porque "el conocimiento científico, como el idioma, es, intrínsecamente, la propiedad común de un grupo, o no es nada en absoluto" Kuhn (1999: 319). A su vez, el indeterminismo o libertad del alumno son los estados del ser del ente o principios del conocimiento que hacen posible estimar que, en la relación de conocimiento, es el sujeto (alumno) el que determina al objeto (conocimiento). Si se supone que, en una relación de conocimiento, el sujeto determina al objeto, entonces, de alguna manera, la facultad cognoscitiva (los sentidos) que permite ver esto se relaciona muy bien con el problema de la relación sujetoobjeto de conocimiento.

\subsubsection{El problema de la percepción de la realidad y las facultades cognoscitivas del sujeto cognoscente.}

Una de las características más importantes del papel del maestro en el proceso enseñanzaaprendizaje, desde la mirada de la teoría humanista aplicada a la educación, consiste en que éste debe ser fidedigno, es decir, real, existente, genuino y puro:

Si puedo crear una relación que, de mi parte, se caracterice por: una autenticidad y transparencia y en la cual pueda yo vivir mis verdaderos sentimientos; una cálida aceptación y valoración de la otra persona como individuo diferente, y una sensible capacidad de ver a mi cliente y su mundo tal como él lo ve. Entonces, el otro individuo experimentará y comprenderá aspectos de sí mismo anteriormente reprimidos; logrará cada vez mayor integración personal y será más capaz de funcionar con eficacia; se parecerá cada vez más a la persona que querría ser; se volverá más personal, más original y expresivo; será más 
emprendedor y se tendrá más confianza; se tornará más comprensivo y podrá aceptar mejor a los demás, y podrá enfrentar los problemas de la vida de una manera más fácil y adecuada (Rogers, 1999, p. 44-45).

Si relacionamos las facultades cognoscitivas del sujeto cognoscente con el problema sobre la percepción de la realidad, es posible observar que el discurso sobre el papel del maestro en el proceso enseñanza-aprendizaje parte de la realidad que existe, se vive, se experimenta, etc. Este tipo de realidad se capta mejor con los sentidos que con la razón, es decir, de manera sensual, porque la verdad significa "adecuación con la realidad... ideas verdaderas son las que podemos asimilar, hacer válidas, corroborar, y verificar; ideas falsas, son las que no" (James, 1975, pp. 156158).

Se sabe que los fenómenos abstractos se captan con la razón, mientras que los concretos con los sentidos. Una de las características más importantes del papel del maestro consiste en que debe ser real, esta característica se trabaja desde lo sensual o sensorial:

...lo importante son las actitudes y sentimientos del terapeuta, y no su orientación teórica: sus procedimientos y técnicas revisten menor importancia que sus actitudes. También merece señalarse el hecho de que, para el cliente, la diferencia reside en la manera en que las actitudes y procedimientos del terapeuta son percibidos, y que esta percepción es fundamental (Rogers, 1999, p. 50).

El humanismo psicológico aplicado a la educación aprecia el papel del maestro en el proceso enseñanza aprendizaje de manera sensual:

¿Cómo puedo ser para que el otro me perciba como una persona digna de fe, coherente y segura, en sentido profundo? He llegado a comprender que ganar la confianza del otro no exige una rígida estabilidad, sino que supone ser sincero y auténtico. He escogido el término coherente para describir la manera de ser que me gustaría lograr (Rogers, 1999, p. 55).

En esta teoría psicológica aplicada a la educación, el papel del maestro se construye a partir del realismo filosófico:

...mis colegas y yo hemos señalado tres actitudes o maneras de ser especialmente importantes en la relación terapéutica. La primera de ellas es la veracidad o autenticidad del terapeuta: que sea lo que parece ser, es decir, que su ser interior y su exterioridad estén en consonancia. La segunda es una atención no posesiva ni juzgadora, forma esta del afecto que crea una atmósfera de seguridad para la persona que busca ayuda. La tercera es la capacidad del terapeuta de escuchar de manera especialmente empática que conduzca a una aceptable comprensión del mundo interior del cliente (Rogers y Freiberg, 1996, p. 88).

Relacionar las facultades cognoscitivas sensuales del sujeto cognoscente con el problema de la percepción de la realidad, con conocimiento o no de este hecho, hace posible construir discursos como el siguiente: 
Cuando el facilitador es una persona auténtica, obra según es y traba relación con el estudiante sin presentar una máscara o fachada, su labor será proclive a alcanzar una mayor eficiencia. Eso significa que tiene conciencia de sus experiencias, que es capaz de vivirlas y de comunicarlas si resulta adecuado. Significa que va al encuentro del alumno de una manera directa y personal, estableciendo una relación de persona a persona. Significa que es él mismo, que no se niega (Rogers y Freiberg, 1996, pp. 185-186).

El maestro debe ser sincero, genuino, transparente, coherente; es decir, real: "la actitud más importante entre las mencionadas es la autenticidad” (Rogers y Freiberg, 1996, p. 190). Los fenómenos concretos se aprecian con los sentidos del sujeto cognoscente: "Cuando un facilitador crea, aunque sea en menor escala, un clima de autenticidad, aprecio y empatía en la clase, entonces descubre que ha iniciado una revolución educativa” (Rogers y Freiberg, 1996, p. 182).

Es necesario aclarar que la realidad concreta se conoce porque impacta nuestros sentidos: "Cuando los clientes de cualquier terapia psicológica valoran en un grado alto a los psicólogos por su autenticidad, excelencia y comprensión empática, es debido a que se ha facilitado el autoaprendizaje y el cambio terapeuta" (Rogers y Freiberg, 1996, p. 193).

Construir el papel del maestro a partir de los sentidos hace posible llegar a estas conclusiones sobre el mismo:

Es posible describir esas actitudes que parecen promover el aprendizaje. En primer lugar, está la autenticidad transparente del facilitador, su voluntad de mostrarse como persona, de ser y vivir la sensaciones y pensamientos del momento. Si esta autenticidad va acompañada del aprecio, confianza y respeto por el alumno, el clima para el aprendizaje se engrandece. Y si a todo esto se agrega una atención empática y sensitiva del alumno, se crea realmente un clima liberador que estimula un aprendizaje autoiniciado y el crecimiento. Se confía en que el estudiante puede evolucionar (Rogers y Freiberg, 1996, p. 198).

Privilegiar lo sensual sobre lo racional en la construcción del discurso sobre el papel del maestro en el proceso enseñanza-aprendizaje permite optar por el realismo y no por el idealismo para proponer las características del papel del maestro en el proceso enseñanza-aprendizaje:

Hay tres condiciones esenciales para que el maestro facilite el aprendizaje y todas se relacionan con su forma se ser. La primera es la de comportarse auténticamente en el sentido de mostrarse ante los alumnos tal como es sin poses o artificios; esto es, manifestar sus sentimientos, sean estos positivos o negativos, sin tratar de negarlos o reprimirlos sino asumirlos (Guzmán y Hernández, 1993, p. 48).

La investigación muestra que cuando un profesor es "real, comprensivo y afectuoso, los alumnos aprenden más acerca de las cuestiones 'básicas', y, asimismo, exhiben la mayor creatividad y capacidad para resolver problemas" (Rogers y Freiberg, 1996, p. 27).

Es posible concluir, en este caso, que el problema de la percepción de la realidad se encuentra directamente relacionado con las facultades cognoscitivas del sujeto cognoscente (los sentidos) en la construcción del mapeo sobre el papel del maestro en el proceso enseñanza-aprendizaje, en el entendido de que se está promoviendo lo real, auténtico, transparente, sensual, empático, 
procedimental, afectivo, comprensivo, respeto, confianza, veracidad, no juzgar, sinceridad, entre otras cualidades del profesor, y se descuida lo ideal que, desde otras teorías psicológicas aplicadas a la educación, se promueven, por ejemplo: el conductismo.

\subsubsection{El problema de la composición de la realidad y las facultades cognoscitivas del sujeto cognoscente.}

En la elaboración de la propuesta sobre el papel del maestro desde el humanismo psicológico aplicado a la educación, es posible observar que se estima que la realidad educativa es una sola compuesta por fenómenos que interactúan entre sí en el tiempo, espacio y circunstancias:

el terapeuta debe experimentar un interés cálido hacia el cliente...Implica tanto la aceptación de las expresiones de sentimientos 'malos', dolorosos, temerosos, anormales por parte del cliente, como de los sentimientos 'buenos', positivos, maduros, seguros y sociables. Supone la aceptación del cliente y la preocupación por él como persona diferente, la aceptación de sus propios sentimientos y experiencias y de los significados personales que distribuye a estos últimos (Rogers, 1999, p. 250).

Si relacionamos el problema de la composición de la realidad con las facultades cognoscitivas del sujeto cognoscente, es posible apreciar que el papel del maestro se construye de manera sensual y se supone que se compone de un todo integrado cuyas partes, en su interacción, se encuentran en tensión unas con otras:

...el asesor debe experimentar una comprensión precisa y empática del cliente, tal como éste lo ve desde su propio interior. La empatía, condición esencial de la psicoterapia, supone sentir el mundo privado del cliente como si fuera el propio, pero sin perder en ningún momento la cualidad de 'como sí' (Rogers, 1999, p. 250).

Se debe suponer que el bien y el mal, en sí mismos, no existen. Se estima que lo que existe son una serie de fenómenos interactuando unos con otros en tiempo, espacio y circunstancias y que, al hacerlo chocan unos con otros y se origina una serie de tensiones entre ellos que producen consecuencias positivas para unos, negativas para otros. Lo que permite pensar que todo depende del lugar en el que se ubique el profesor con respecto a los demás componentes de la estructura total del proceso enseñanza-aprendizaje. El profesor debe: “crear un clima de aceptación, estima y confianza en el salón de clase de tal manera que exista un mutuo respeto entre profesores y alumnos" (Guzmán y Hernández, 1993, p. 48). Es decir, debe colocarse en buenos términos con relación a las demás partes que interactúan en la estructura del proceso enseñanza-aprendizaje.

\subsubsection{El problema de la clasificación del conocimiento y las facultades cognoscitivas del sujeto cognoscente.}

Toda clasificación sobre cualquier concepto, idea, noción o categoría, es arbitraria y obedece los intereses y necesidades de quien realiza el mapeo sobre la misma. Para fines de este trabajo y, en el entendido de que se supone que todo sujeto cognoscente cuenta sólo con dos facultades cognoscitivas (la razón y los sentidos) para resolver los problemas del conocimiento, ya se hizo notar en el apartado correspondiente al problema del origen del conocimiento que existen cuatro posibilidades para resolverlo: estimar que se origina en la razón, en los sentidos, que comienza en la razón y termina en los sentidos o que inicia en los sentidos y termina en la razón. 
La psicología humanista aplicada a la educación, estima que se aprende por experiencia, es decir, es de la opinión de que "aprender consiste en plantear mis propias incertidumbres, tratar de esclarecer mis dudas y acercarme al significado real de mi experiencia” (Rogers, 1999, p. 244).

El término 'experiencia' implica práctica, uso, estilo o rutina. Es decir, el alumno aprende lo que vive, siente, sufre, experimenta, entre otras cosas. Aprender significa "dejarme llevar por mi experiencia, en un sentido que parece ser progresivo, hacia objetivos que apenas pueda discernir, mientras trato de comprender al menos el sentido básico de esa experiencia" (Rogers, 1999, p. 244).

Si se relacionan la facultad cognoscitiva del sujeto cognoscente del humanismo aplicado a la educación con el problema de la clasificación del conocimiento, es posible apreciar que la primera es causa del segundo. Es decir, se puede opinar que el conocimiento es intuitivo o experiencial porque se supone que se aprende por experiencia; o sea, se estima que el conocimiento es de tipo sensual, experiencial, intuitivo, vivencial, entre otros adjetivos que se le pueden agregar a este tipo de conocimiento.

Debido a ello se promueve un maestro comprensivo, empático, entre otras características, que permitan facilitar el aprendizaje:

Hay tres condiciones esenciales para que el maestro facilite el aprendizaje y todas se relacionan con su forma se ser... La última condición es la de tener una comprensión empática; lo que significa ponerse en el lugar de los alumnos -colocarse en sus zapatostratando de comprender sus reacciones íntimas (Guzmán y Hernández, 1993, p. 48).

Es posible concluir, en este caso, que el problema de la clasificación del conocimiento se encuentra directamente relacionado con las facultades cognoscitivas del sujeto cognoscente (los sentidos) en la construcción del mapeo sobre el papel del maestro en el proceso enseñanza-aprendizaje, en el entendido de que se está promoviendo lo sensual, intuitivo o irracional y se descuida lo racional, que, desde otras teorías psicológicas aplicadas a la educación se promueve, por ejemplo: el conductismo, como ya se hizo mención.

\subsubsection{El problema dell concepto y criterio de verdad del conocimiento y las facultades cognoscitivas del sujeto cognoscente.}

Si la psicología humanista aplicada a la educación se adhiere a la opinión de que el conocimiento es de tipo intuitivo o sensual, entonces, también es posible que el concepto de verdad que se aplica para generar ese tipo de conocimiento provenga también de los sentidos:

si el asesor es coherente, de manera tal que sus palabras concuerden con sus sentimientos; si manifiesta una aceptación incondicional por el cliente y comprende los sentimientos esenciales de este último tal como él los ve, entonces existe una gran probabilidad de lograr una relación de ayuda efectiva (Rogers, 1999, p. 54).

Es decir, para la psicología humanista aplicada a la educación, la verdad se puede definir como la concordancia entre el pensamiento y el objeto pensado. 
Si relacionamos las facultades cognoscitivas del sujeto cognoscente con el problema del concepto de verdad de los discursos de la teoría psicológica humanista aplicada a la educación, es posible observar que la verdad se define de manera trascendente, es decir, como la concordancia del pensamiento con el objeto pensado: "pienso que sólo me interesa aprender, incorporar preferiblemente cosas importantes que ejerzan una influencia trascendente sobre mi propia conducta” (Rogers, 1999, p. 244).

El criterio para elaborar el concepto trascendente de verdad se basa en el realismo filosófico:

Pienso que una de mis mejores maneras de aprender -pero también una de las más difícilesconsiste en abandonar mis propias actitudes de defensa, al menos temporalmente, y tratar de comprender lo que la experiencia de la otra persona significa para ella (Rogers, 1999, p. 244).

Ya se indicó que la palabra 'experiencia' indica vivencia, existencia, realidad, entre otros calificativos que se le pueden adherir; luego entonces, es posible establecer una relación de causa-efecto entre los sentidos, como facultad cognoscitiva del sujeto cognoscente de la teoría psicológica humanista aplicada a la educación y el problema del criterio de verdad del conocimiento que se elabora sobre el papel del maestro desde dicha teoría.

Es posible concluir, en este caso, que el problema del concepto y criterio de verdad del conocimiento se encuentra directamente relacionado con las facultades cognoscitivas del sujeto cognoscente (los sentidos) en la construcción del mapeo sobre el papel del maestro en el proceso enseñanza-aprendizaje, en el sentido de que se está promoviendo lo trascendente y se descuida lo inmanente, que, desde otras teorías psicológicas aplicadas a la educación se promueve, por ejemplo: el multicitado conductismo.

En ese entendido, a la facultad cognoscitiva del sujeto cognoscente (los sentidos) corresponde determinada necesidad o intensión del mismo, dependiendo del problema del conocimiento con el que se relacione dicha facultad cognoscitiva.

\section{Conclusiones.}

A lo largo de la investigación fue posible demostrar la hipótesis de que, entre los problemas del conocimiento y las facultades cognoscitivas del sujeto cognoscente de la teoría humanista aplicada a la educación, cuando éste pretende construir el mapeo sobre el papel del maestro en el proceso enseñanza-aprendizaje, se pueden establecer una serie de relaciones:

- Dichas relaciones son causales; es decir, todos y cada uno de los problemas del conocimiento (posibilidad, origen, esencia, clasificación y concepto y criterios de verdad) se relacionan con todas y cada una de las facultades cognoscitivas del sujeto cognoscente (razón, sentidos, razónsentidos, sentidos-razón).

- Las relaciones mencionadas se establecen en términos de los intereses, necesidades o motivos existenciales del sujeto cognoscente (quietud y/o movimiento, orden y/o caos, determinismo y/o libertad, ser y/ o deber ser, unidad y/o dualidad, entre otros); mismos que le conducen a la elaboración de un conocimiento de tipo racional y/o sensual y a unos criterios y conceptos inmanentes y/o trascendentes de verdad. 
- En el caso del humanismo psicológico aplicado a la educación y, concretamente en la construcción que éste pretende realizar del mapeo sobre el papel del maestro en el proceso enseñanza-aprendizaje, se puede observar que todos y cada uno de los mencionados problemas del conocimiento se pretenden resolver con los sentidos.

- La consecuencia de lo anterior consiste en que se privilegia el movimiento o cambio sobre la quietud o calma, el caos o desorden sobre el orden o estabilidad, el indeterminismo o la libertad sobre el determinismo o permanencia, la unidad o inclusión sobre la dualidad o exclusión, lo intuitivo sobre lo racional y lo trascendente sobre lo inmanente.

- La consecuencia necesaria de lo anterior consiste en que el humanismo psicológico aplicado a la educación promueve un papel del maestro en el proceso enseñanza-aprendizaje en términos de desempeñar un papel no directivo, es decir, de concretarse a facilitar las condiciones del aprendizaje para que éste se dé en forma autónoma, partiendo de las potencialidades y necesidades del alumno y comportándose con el mismo de manera realista, incluyente, empática y comprensiva; lo que permitiría establecer relaciones de respeto y fomentar un clima social; que haría posible un aprendizaje lúdico, placentero y libertario; promoviendo relaciones de respeto, apoyo, curiosidad, duda, búsqueda personalizada, entre otras cosas.

La principal limitación de la teoría psicológica humanista aplicada a la educación consiste en que el papel del maestro que se propone es muy importante y deseable en el contexto actual de la educación, no obstante, se descuidan aspectos socioculturales, psicogenéticos, cognoscitivos, psicoanalíticos y conductistas de la educación que son tratados a profundidad por Vygotsky, Piaget, Ausubel, Freud y Skinner, respectivamente, y sus seguidores. Para todos y cada uno de ellos, el papel del maestro es totalmente distinto de la propuesta de la psicología humanista aplicada a la educación. Para Skinner, el papel del maestro debe concretarse en ser un tecnólogo de la educación, director y controlador del proceso de aprendizaje e ingeniero conductual, es decir diametralmente opuesto a la propuesta que se está analizando; para Freud, debe centrarse en lo terapéutico, es decir, fomentar personalidades psicológicamente sanas y favorecer el sano desarrollo psicoemocional del alumno; según Ausubel, se trata de fomentar el desarrollo y práctica de los procesos cognoscitivos del alumno: identificando los conocimientos previos y relacionándolos con los nuevos para lograr el aprendizaje significativo; Piaget, es de la idea de que debe ayudar al educando a construir su propio conocimiento, promover el desarrollo y autonomía del educando y crear una atmósfera de reciprocidad, respeto y autoconfianza; finalmente, de acuerdo con Vygotsky, el maestro debe crear y negociar zonas de desarrollo próximo y ser un experto que guía y mediatiza los saberes socioculturales que se deben aprender e internalizar.

Lo anterior permite estimar la principal deficiencia de este trabajo, faltaría analizar a profundidad el papel del maestro que proponen las teorías aludidas para estar en condiciones de determinar los alcances y limitaciones epistemológicas de todas y cada una de ellas.

Es de considerar que el aporte principal de este estudio a la realidad educativa consiste en la necesidad de formar para la investigación desde la epistemología, entendida como conocimiento del conocimiento, es decir, de comprender los alcances y limitaciones ontológicas y epistemológicas de las teorías de la psicología aplicadas a la educación. 
La prospectiva de este trabajo consiste en la posibilidad e invitación a investigar las relaciones entre las necesidades existenciales, las necesidades ontológicas, los problemas del conocimiento, las facultades cognoscitivas con las que se pretenden resolver los problemas del conocimiento, los supuestos filosóficos en los que - de manera necesaria- se ubica el sujeto cognoscente al pretender resolver los problemas del conocimientos de una u otra manera y, finalmente, el discurso educativo, cuando se pretende explorar, describir, explicar, interpretar o comprender el papel del maestro, el concepto de aprendizaje, la motivación, la metodóloga de la enseñanza, la evaluación, el concepto de alumno, las metas de la educación, entre otros fenómenos del hecho educativo. Es decir, fomentar la posibilidad de hacer realidad el sueño kantiano en el campo educativo, quien entendía la ilustración como la posibilidad de liberación del hombre de su culpable incapacidad.

\section{Referencias}

Descartes, R. (2018). Las pasiones del alma. Lectorum.

Guzmán, J. C., \& Hernández Rojas, G. (1993). Implicaciones educativas de seis teorías psicológicas. CONALTE. Hessen, J. (1999). Teoría del Conocimiento. Porrúa

Kant, I. (1994). Filosofía de la historia. FCE.

Kuhn T., S. (1999). La Estructura de las Revoluciones Científicas. Fondo de Cultura Económica.

Nietzsche, F. (1976). Más allá del bien y del mal. Editores Unidos Mexicanos

Ortega y Gasset, J. (1998). ¿Qué es filosofía? Porrúa

Polo, L. (2006). Curso de teoría del conocimiento. Tomo I. Ediciones Universidad de Navarra

Platón (2018). Diálogos. Editorial Porrúa

Rogers, C. (1991). El proceso de convertirse en persona. Paidós

Rogers, C., \& Freiberg, J. (1996). Libertad y creatividad en la educación. Paidós

Schopenhauer, A. (1997). El Mundo como Voluntad y Representación. Porrúa

Spinoza, B. (1990). Ética. Porrúa

Verneaux, R. (2011). Curso de filosofía tomista. Epistemología general o crítica del conocimiento. Herder.

William, J. (1975). Pragmatismo. Colofón.

\section{AUTOR}

José Nava. Doctor en Educación por la Universidad Alfa-Lambda. Doctor y Maestro en Derecho por la Universidad Autónoma del Estado de México. Docente Investigador del Instituto Superior de Ciencias de la Educación del Estado de México.

\section{Conflicto de intereses}

El autor informa que no existe conflicto de interés posible.

Financiamiento

Artículo financiado por el Instituto Superior de Ciencias de la Educación del Estado de México. No hubo asistencia financiera de partes externas al presente artículo.

Agradecimientos

$$
\mathrm{N} / \mathrm{A}
$$

Nota

El artículo es producto del proyecto: “Los principios de la ciencia y el discurso educativo: sus relaciones ontoepistemológicas" 